\title{
High Performance Nanostructured Silicon-Organic Quasi $p$ - $n$ Junction Solar Cells via Low-Temperature Deposited Hole and Electron Selective Layer
}

Yuqiang Liu', Zhi-guo Zhang ${ }^{2}$, Zhouhui Xial, Jie Zhang ${ }^{3}$, Yuan Liul, Feng Liang ${ }^{1}$, Yongfang Li ${ }^{2}$, Tao Song ${ }^{1}$, Xuegong Y ${ }^{4}$, Shuit-tong Lee ${ }^{1}$, Baoquan Sun ${ }^{1}$ *

1 Jiangsu Key Laboratory for Carbon-Based Functional Materials and Devices, Institute of Functional Nano \& Soft Materials (FUNSOM), Soochow University, Suzhou 215123, China

${ }^{2}$ Beijing National Laboratory for Molecular Sciences, CAS Key Laboratory of Organic Solids, Institute of Chemistry, Chinese Academy of Sciences, Beijing 100190, China.

${ }^{3}$ Department of Electronic Engineering, the Chinese University of Hong Kong, New Territories, Hong Kong

${ }^{4}$ State Key Laboratory of Silicon Materials and Department of Materials Science and Engineering, Zhejiang University, Hangzhou 310027, China

Email: bqsun@suda.edu.cn. Tel: (86)-512-65880820. Fax: (86)-512-65882846

\section{Calculation the quasi $p-n$ junction emitter layer parameters}

For quasi $p$ - $n$ junction device, majority carrier concentration in emitter layer was calculated from equation (1) and minority carrier diffusion length was calculated from equation (2) and (3). ${ }^{2,3}$

$$
\begin{array}{r}
V_{b i}=\frac{k T}{q} \ln \left(\frac{N_{A} N_{D}}{n_{i}{ }^{2}}\right) \\
\mathrm{L}=\sqrt{D \tau} \\
\mathrm{D}=\frac{k T}{q} \mu
\end{array}
$$

Here, the data of $\mathrm{N}_{\mathrm{D}}$ was chosen of $1 \times 10^{17} / \mathrm{cm}^{3}$, the minority carrier (electron) mobility $\mu_{\mathrm{n}}=\sim 800 \mathrm{~cm}^{2} \mathrm{~V}^{-1} \mathrm{~s}^{-1}$, minority carrier (electron) lifetime $\tau=\sim 1 \times 10^{-5} \mathrm{~s}^{2,3}$

Majority carrier concentration in emitter layer

$$
N_{A}=e^{\frac{q V_{b i}}{k T}} \times \frac{n_{i}{ }^{2}}{N_{D}} \approx 2.6 \times 10^{17} / \mathrm{cm}^{3},
$$

Minority carrier diffusion length in emitter layer 


$$
\mathrm{L}=\sqrt{D \tau}=\sqrt{\frac{k T}{q} \mu_{n} \tau} \approx 141 \mu \mathrm{m} .
$$




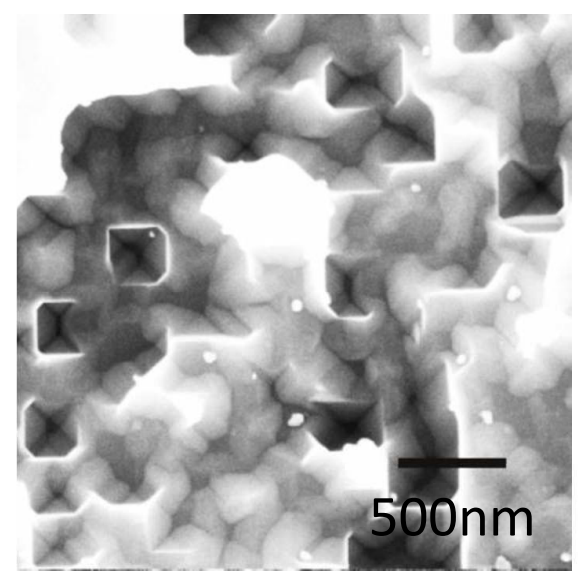

Figure S1. SEM image of nanostructured silicon fabricated by MIAE and THAM post-treatment. 


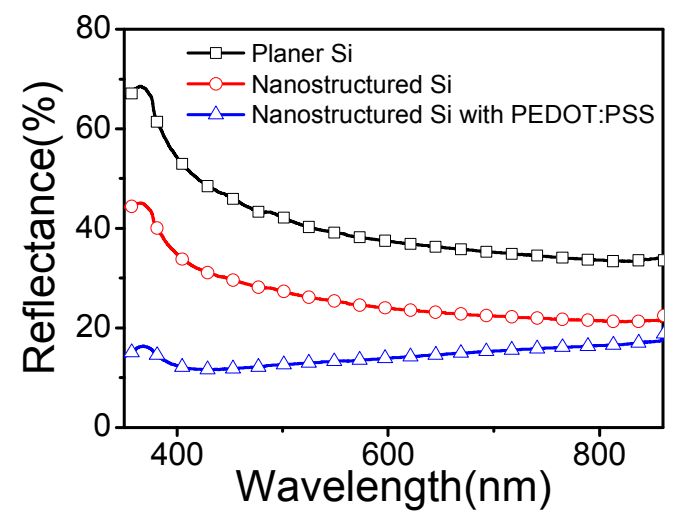

Figure S2. Reflection spectra of planer silicon, nanostructured silicon and nanostructured silicon with PEDOT:PSS. 
(a)

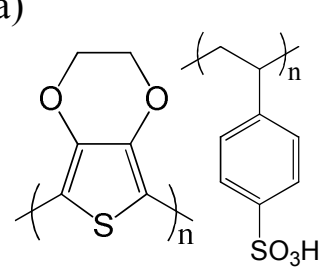

(b)

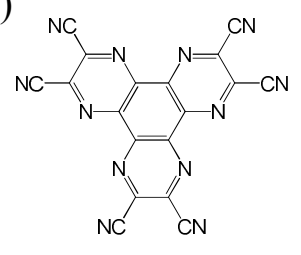

(c)

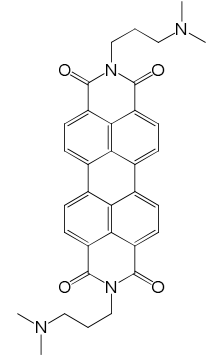

Figure S3. Chemical structures of (a) PEDOT:PSS; (b) HAT-CN; (c) PDIN. 
(a)

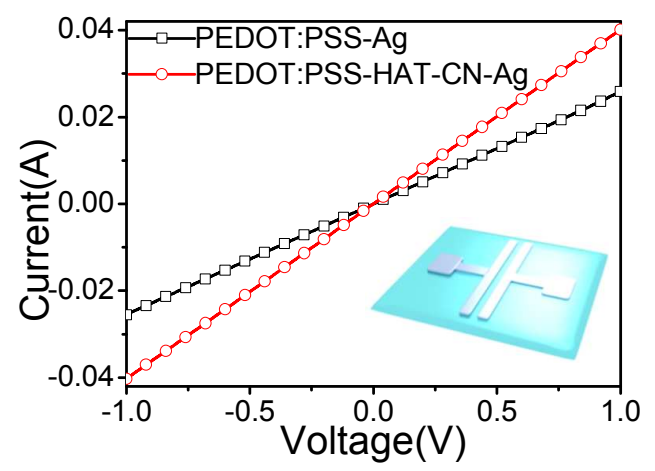

(b)

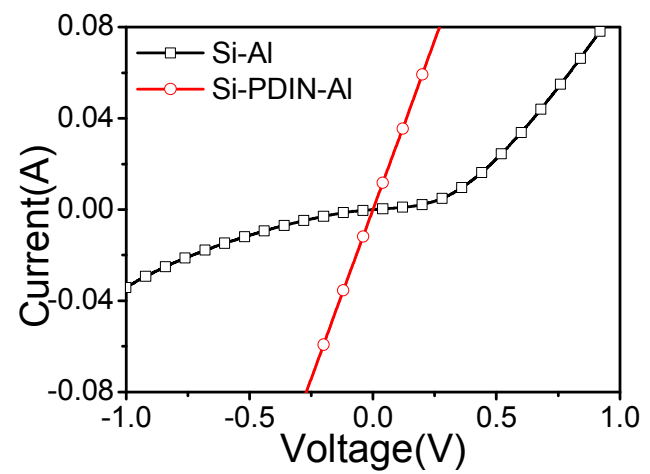

(c)

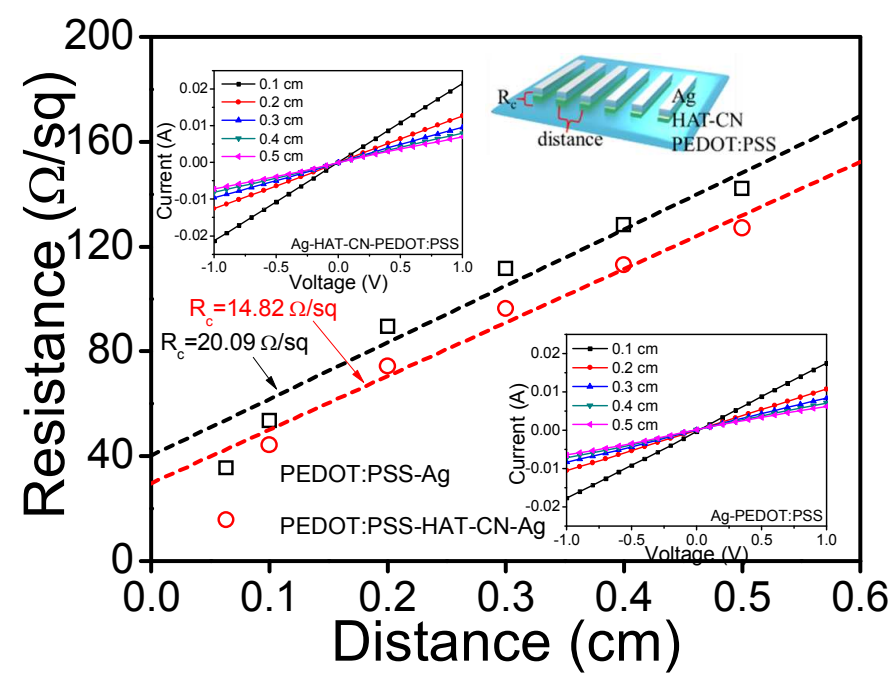

Figure S4. (a) Current-voltage curves between the T-shaped Ag pads deposited onto PEDOT:PSS with and without HAT-CN on silicon; inset was T-shaped pattern of Al or Ag pads on substrate; (b) Current-voltage curves between the T-shaped Al pads deposited onto backside of silicon with and without PDIN. (c) Contact resistance between PEDOT:PSS and Ag with and without HAT-CN layer at different Ag grid distance; Insets showed the current-voltage measurements at different interval distance without and with HAT-CN. The scheme for measured contact resistance with different distances was also in inset. 
(a)

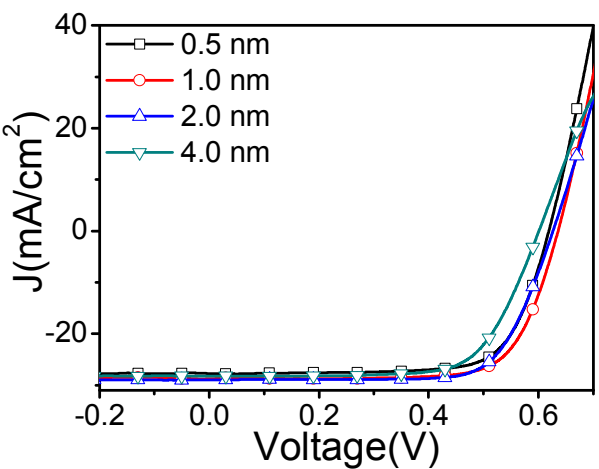

(c)

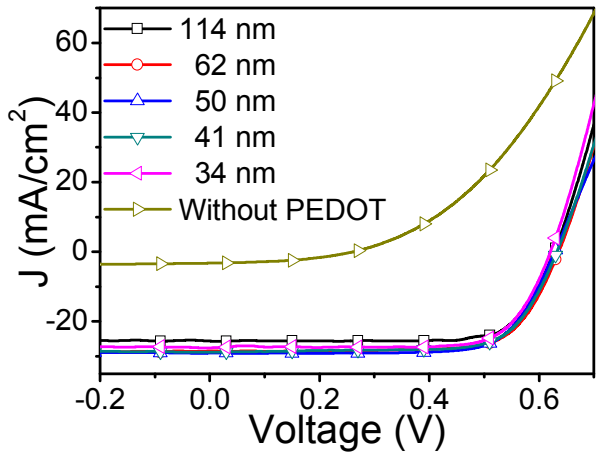

(b)

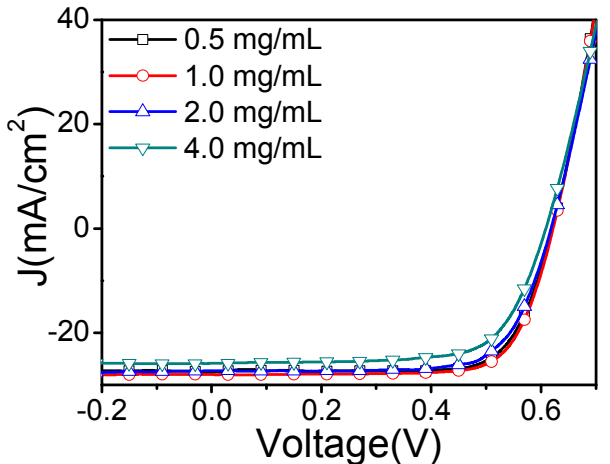

(d)

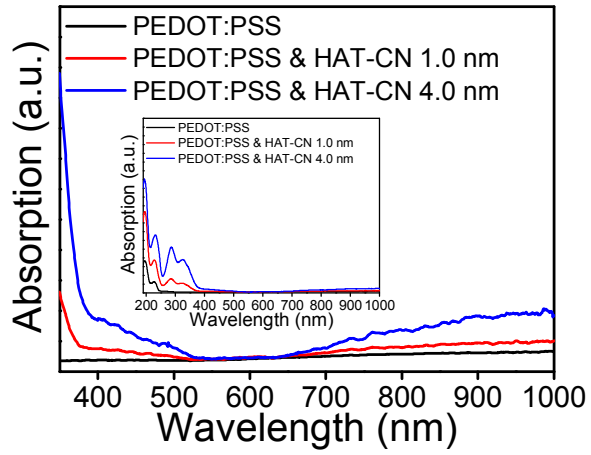

Figure S5. Current density-voltage curves under illumination of AM 1.5 of solar cells. The device with (a) different thickness of HAT-CN (Ag(grid)/HAT-CN/PEDOT:PSS/Si/Al), (b) different thickness (or concentration) of PDIN (Ag(grid)/PEDOT:PSS/Si/PDIN/AI) and (c) with different PEDOT:PSS thickness based on structure of $\mathrm{Ag}$ (grid)/HAT-CN/PEDOT:PSS/Si/Al; (d) Absorption spectra of PEDOT:PSS film with different HAT-CN thickness; inset was full absorption spectra range. 

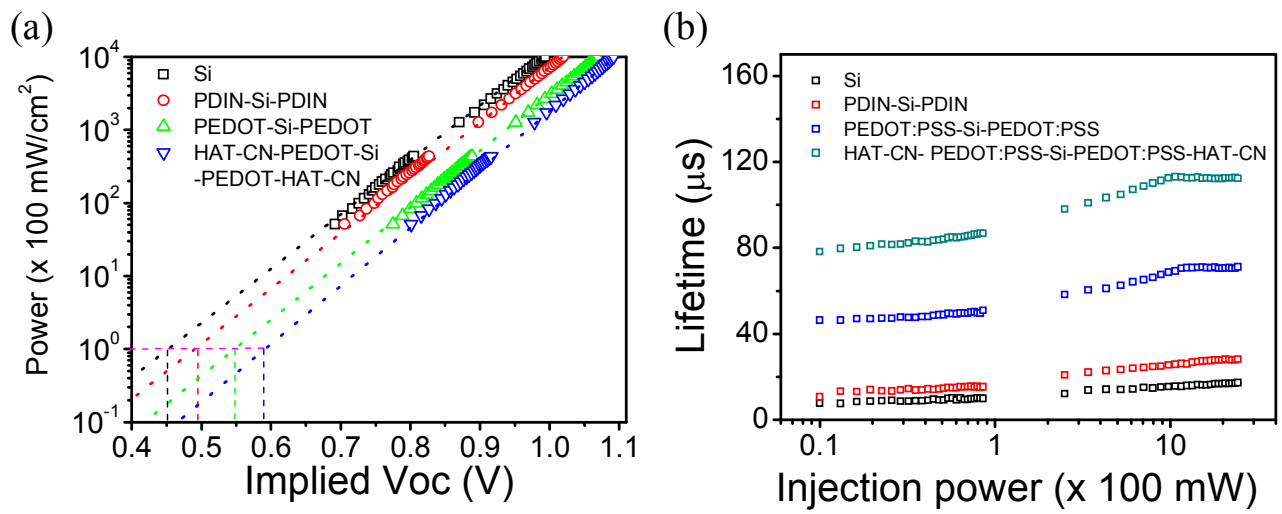

Figure S6. (a) Implied open circuit voltage, (b) Minority carrier lifetime vs. illumination intensity by laser diode with wavelength of either $978 \mathrm{~nm}$ or $980 \mathrm{~nm}$. The horizontal and vertical dashed lines showed the implied open circuit voltages at one sun in (a). The low and high intensity range is generated from a $978 \mathrm{~nm}$ laser diode and $980 \mathrm{~nm}$ one, respectively. The silicon substrates are symmetrical structure of PDIN/Si/PDIN, PEDOT:PSS/Si/PEDOT:PSS, HAT-CN/PEDOT:PSS/Si/PEDOT:PSS/HAT-CN. Here, the substrates are silicon with ( $<100>$-oriented, $300 \mu \mathrm{m}, 1 \sim 3 \Omega / \mathrm{sq})$. 
Table S1. Typical silicon solar cell emitter parameters.

\begin{tabular}{ccc}
\hline Emitter & P-N Junction ${ }^{\text {a) }}$ & Quasi P-N Junction $^{\text {b) }}$ \\
\hline Thickness/nm & 500 & 106 \\
Majority carrier concentration in emitter layer $/ \mathrm{cm}^{-3}$ & $1 \times 10^{19}$ & $2.6 \times 10^{17}$ \\
Minority carrier concentration in emitter layer $/ \mathrm{cm}^{-3}$ & 10 & $3.8 \times 10^{2}$ \\
Minority carrier diffusion length in emitter layer $/ \mu \mathrm{m}$ & 14 & 141 \\
EQE $(@ 365 \mathrm{~nm}) / \%$ & $50^{1}$ & 65 \\
\hline
\end{tabular}

a): Parameters of traditional P-N junction are from Reference 1 and 2.

b): Ag(grid)/HAT-CN/PEDOT:PSS/Si/PDIN/Al (PDIN \& HAT-CN), room-temperature. 
Table S2. Average electrical output characteristics for the devices with different structures.

\begin{tabular}{ccccc} 
Device Structure $^{\text {a) }}$ & $\mathbf{V}_{\mathbf{o c}}(\mathbf{m V})$ & $\mathbf{J}_{\mathbf{s c}}\left(\mathbf{m A} / \mathbf{c m}^{\mathbf{2}}\right)$ & $\mathbf{F F}$ & PCE (\%) \\
\hline Reference & $597 \pm 9.6$ & $26.47 \pm 0.13$ & $0.696 \pm 0.010$ & $10.98 \pm 0.095$ \\
PDIN & $622 \pm 3.0$ & $28.27 \pm 0.35$ & $0.735 \pm 0.011$ & $12.97 \pm 0.046$ \\
HAT-CN & $628 \pm 3.2$ & $28.71 \pm 0.19$ & $0.740 \pm 0.002$ & $13.37 \pm 0.045$ \\
PDIN \& HAT-CN & $631 \pm 1.9$ & $29.46 \pm 0.26$ & $0.758 \pm 0.002$ & $14.03 \pm 0.061$
\end{tabular}

a): $\mathrm{Ag}$ (grid)/PEDOT:PSS/Si/Al (reference), $\mathrm{Ag}$ (grid)/PEDOT:PSS/Si/PDIN/Al (PDIN), Ag(grid)/HAT-CN/PEDOT:PSS/Si/Al (HAT-CN), Ag(grid)/HAT-CN/PEDOT:PSS/Si/PDIN/Al (PDIN \& HAT-CN) 
Table S3. Electrical output characteristics for the devices with different PEDOT thickness.

\begin{tabular}{ccccc} 
PEDOT Thickness $^{\text {a) }}$ & $\mathbf{V}_{\mathbf{o c}}(\mathbf{m V})$ & $\mathbf{J}_{\mathbf{s c}}\left(\mathbf{m A} / \mathbf{c m}^{2}\right)$ & FF & PCE (\%) \\
\hline $114 \mathrm{~nm}$ & 627 & 25.62 & 0.771 & 12.25 \\
$62 \mathrm{~nm}$ & 636 & 28.50 & 0.744 & 13.36 \\
$50 \mathrm{~nm}$ & 628 & 29.11 & 0.745 & 13.44 \\
$41 \mathrm{~nm}$ & 632 & 28.78 & 0.728 & 13.21 \\
$34 \mathrm{~nm}$ & 621 & 27.57 & 0.743 & 12.71 \\
Without PEDOT & 260 & 3.24 & 0.435 & 0.37 \\
\hline
\end{tabular}

a): Ag(grid)/HAT-CN/PEDOT:PSS/Si/Al

\section{REFERENCES}

1. Zhao, J.; Wang, A.; Green, M. A.; Ferrazza, F. $19.8 \%$ Efficient "Honeycomb" Textured Multicrystalline and 24.4\% Monocrystalline Silicon Solar Cells. Appl. Phys. Lett. 1998, 73, 1991-1993.

2. Nelson, J. The Physics of Solar Cells. World Scientific: 2003.

3. Sze, S. M. Semiconductor Devices: Physics and Technology. John Wiley \& Sons: 2008. 